\title{
Use of laryngeal mask airway in a patient with a large tongue cyst: A case report
}

\author{
Monica Tan ${ }^{1 *}$ and Yee Yian Ong ${ }^{2}$ \\ ${ }^{1}$ Resident, Department of Anaesthesiology, Singapore General Hospital, Singapore \\ ${ }^{2}$ Associate Consultant, Department of Anaesthesiology, Singapore General Hospital, Singapore
}

\begin{abstract}
Patients with intra-oral lesions undergoing surgery often pose airway challenges to the anaesthetist. Successful airway management in such patients requires recognition of a potential airway problem. Intubation is often described in patients with large oral lesions undergoing surgery, but there is little mention on the use of supraglottic airway devices. Most of the reports are also in the paediatric population where intra oral lesions are most commonly encountered. Here we describe our experience with the use of a Laryngeal Mask Airway (LMA) in a 75-year-old woman with a large anterior tongue cyst undergoing wide excision of a malignant melanoma on her forearm with a split skin graft creation.
\end{abstract}

\section{Introduction}

A patient with a markedly enlarged tongue presents a unique challenge to anaesthetists. There are several reports on anaesthetic management in macroglossia [1-4], but these are largely in paediatric populations where congenital malformations of the tongue are most encountered. The surgeries performed in these cases often involve the tongue lesion itself and difficult airway management is often described with successful intubation as the end point. There is limited literature available on the airway management in an adult with a tongue mass, undergoing non-oral surgery. We describe a case of a large anterior tongue cyst in a 75 year old woman who had presented for excision biopsy of malignant melanoma of the forearm with a split skin graft.

\section{Case report}

A 75-year-old woman, who was recently diagnosed with a left forearm dorsum melanoma, was scheduled for wide excision of the melanoma, sentinal lymph node biopsy, and a split skin graft. Her medical issues included hypertension and hyperlipidemia for which she was on medications. Of particular interest was a tongue cyst she had been diagnosed with 2 years ago for which she declined surgery.

She presented to the Preoperative Evaluation Clinic 10 days prior to her operation where examination of the oral cavity revealed a large anterior tongue mass measuring $4 \mathrm{~cm} \mathrm{x} 4 \mathrm{~cm} \mathrm{x} 3 \mathrm{~cm}$ (Figures 1 and 2). The lesion was pink in colour, fluctuant and not tender to touch, with a smooth surface and soft consistency. The patient verbalised that the tongue mass had been present for 15 years and had gradually grown in size. 2 years ago it had been seen by an Otolaryngologist who diagnosed it as a benign cyst. She was offered surgery but had declined. There was no history of respiratory difficulty, snoring, dysphagia, trauma, pain, or bleeding from the mass. The patient was edentulous and had a Mallampati score of 2, with a mouth opening of 3 finger breadths $(5 \mathrm{~cm})$, and a thyromental distance of 4 finger breadths $(6 \mathrm{~cm})$. Her systemic examination revealed no abnormalities.

The patient was referred to an otolaryngologist prior to her operation to evaluate for other masses in the airway. Nasoendoscopy was performed which revealed no other masses. The patient was offered intervention to remove or reduce the size of the cyst prior to her operation but she declined.

On the day of surgery, the anaesthetic team decided to proceed with general anaesthesia using an LMA. A difficult airway cart was prepared and suction was kept ready should the cyst rupture on LMA insertion. Monitoring was initiated with electrocardiography, arterial saturation, and non invasive blood pressure monitoring. Preoxygenation was initiated using a large facemask and the patient was induced with Propofol. Bag mask ventilation was easy. A Proseal LMA, size 3, lubricated with a water-based gel, was easily inserted at the first attempt using the digital technique. The head was placed in a neutral position, proper positioning of the device was confirmed by capnography, and the LMA was secured with tape (Figure 3 and 4).

Anesthesia was maintained with Propofol targeted concentration infusion under spontaneous ventilation. Under spontaneous ventilation, the patient achieved adequate tidal volumes of $300 \mathrm{ml}$ with peak inspiratory pressures of $2 \mathrm{mmHg}$. The surgeons proceeded with surgery uneventfully - the left forearm melanoma was excised, sentinal lymph nodes from the left axilla were removed, and a split skin graft from the right thigh was applied over the left forearm wound. The LMA was successfully removed in the operating theatre at the end of surgery, it had been insitu for a total of 1 hour. The post operative period was uneventful and the patient was returned to the ward fully awake and comfortable.

Correspondence to: Monica Tan, Resident, Department of Anaesthesiology, Singapore General Hospital, 63 Sunset Way \#04-09, Singapore 597088, Tel: +65 98369174; E-mail: monica.tan@mohh.com.sg

Key words: difficult airway, laryngeal mask airway (LMA), oral lesion, tongue cyst Received: April 05, 2015; Accepted: May 07, 2015; Published: May 10, 2015 


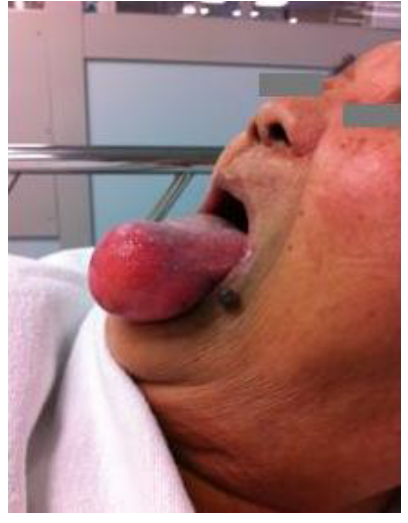

Figure 1.

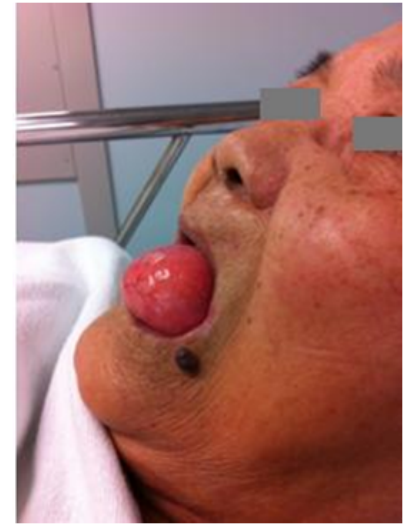

Figure 2.

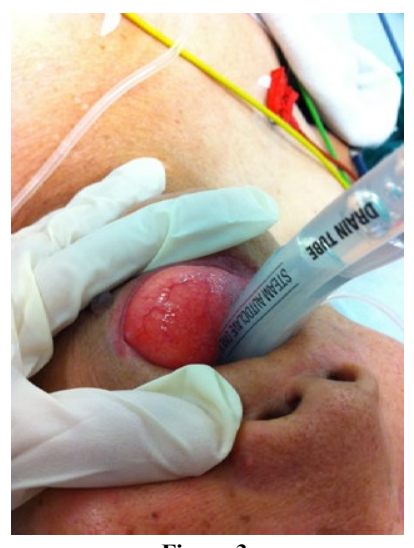

Figure 3.

\section{Discussion}

In existing case reports of airway management in patients with macroglossia, intubation is often the aim. Options for this potentially difficult intubation include the use of a fibreoptic bronchoscope [5], and awake intubation is commonly performed to avoid the risk of airway compromise and difficult mask ventilation after induction of general anaesthesia. In preoperative evaluation, the history is often used to estimate the degree of difficulty in facemask ventilation. If a patient does not snore or experience obstructive sleep apnea, it is likely that his airway will remain patent during anaesthesia. If the history indicates

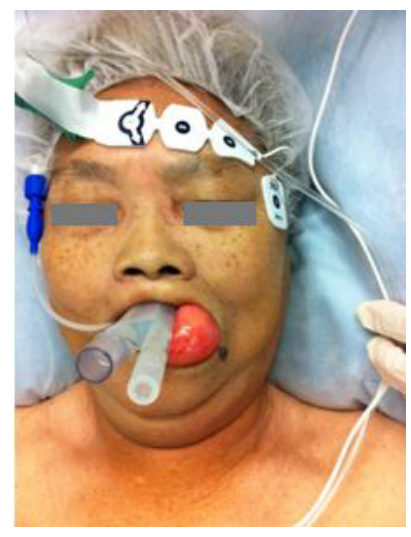

Figure 4.

a lack of airway patency during sleep, however, successful facemask ventilation is unlikely and an awake fibreoptic intubation should be used [6]. The invaluable advantage of awake fibreoptic intubation is its safety. However, it requires patient cooperation and may often be distressing to undergo. The use of Glidescope or blind nasotracheal intubation, are other useful techniques in such scenarios [7,8]. A Glidescope allows minimal manipulation of the airway to visualise the epiglottis and glottis opening and hence induce less trauma on a large tongue mass. Blind nasotracheal intubation would also bypass a large tongue, though several attempts may be required to achieve adequate intubation.

In our case, we considered the use of an LMA as it was a short operation that did not involve the oral cavity. The patient's tongue cyst, though big, occupied only the anterior $1 / 3$ of her tongue. She was also edentulous and had adequate mouth opening. These factors were reassuring that once the laryngeal mask airway was inserted past the anterior tongue, it would enter the pharynx fairly smoothly. The use of a supraglottic device would also be a less traumatic option as it obliterates the need to use a laryngoscope, as in the case of intubation. A laryngoscope could exert excessive pressure on the tongue cyst, increasing the risk of rupture and aspiration of cyst contents. That said, laryngoscope aided tongue elevation in difficult LMA insertions have been described, though the goal in those scenarios was soft-handed tongue elevation only without the need for complete visualisation of the laryngeal opening [9].

The use of an LMA also avoids the use of a muscle relaxant during induction as use of a muscle relaxant during induction of anesthesia may result in a situation where it is both difficult to ventilate and intubate, possibly warranting the creation of a surgical airway rapidly. Hence, maintenance of spontaneous breathing allows a way out, should there be difficulty in securing the airway.

On removal of the LMA, a nasopharyngeal tube was kept readily available as there was a concern that the patient, still drowsy, may have upper airway obstruction from her large tongue mass. In such cases, pre-operatively, it is important to find out from the patient or family members if there are any respiratory difficulties when the patient is asleep, and if so, if there are particular positions that the patient prefers to sleep in to overcome his breathing difficulties.

Lingual nerve injury with the optimal use of a Proseal LMA has been reported and is most likely due to pressure neuropraxia from the LMA tube [10]. The lingual nerve is at risk of compression as it enters the mouth below the inferior border of the superior constrictor and continues against the periosteum of the mandible posterior to the third 
molar. This was of particular concern in our patient as the presence of a large tongue mass increases the risk of excessive intraoral pressures and hence compressive neuropraxia. However, the patent was reviewed 24 and 48 hours post operatively and had no decreased sensation, loss of taste, or pain over her tongue.

\section{Conclusion}

A thorough preop evaluation is essential in the anaesthetic management of a patient with a tongue mass going for surgery. History taking should pay particular attention to eliciting signs and symptoms of upper airway obstruction. A review by an otolaryngologist to exclude concurrent airway lesions is also important. These preoperative assesments allow the anaesthetist to anticipate the level of difficulty in managing the airway. Should the operation be relatively short and not involve the oral cavity, with little signs and symptoms from the patient to suggest airway obstruction, the use of an LMA should be considered. Regardless of whether a supraglottic device is deemed suitable or not, equipment for a difficult airway should always be made readily available.

\section{Acknowledgements}

We would like to thank the patient in the case study for consenting to having her case reported and photographs taken for this case report.

\section{References}

1. Xue FS, He N, Xu YC, Liao X (2009) Anesthesia and airway management for children with macroglossia. Paediatr Anaesth 19: 275-277. [Crossref]
2. Goktas U, Tekin M, Kati I, Toprak K, Yüce HH (2008) Difficult airway management in lymphangiomatos macroglossia. Paediatr Anaesth 18: 1127-1128. [Crossref]

3. Skelton VA, Goodwin A (1999) Perinatal management of a neonate with airway obstruction caused by rhabdomyosarcoma of the tongue. Br J Anaesth 83: 951-955. [Crossref]

4. Thomas ML, McEwan A (1998) The anaesthetic management of a case of Kawasaki's disease (mucocutaneous lymph node syndrome) and Beckwith-Weidemann syndrome presenting with a bleeding tongue. Paediatr Anaesth 8: 500-502. [Crossref]

5. Chhaya J, et al. (2011) Anaesthetic considerations in macroglossia due to haemangioma of the tongue: a case report. Journal of Clinical and Diagnostic Research 5: 840-842.

6. Nargozian C (2004) The airway in patients with craniofacial abnormalities. Paediatr Anaesth 14: 53-59. [Crossref]

7. Cooper RM, Pacey JA, Bishop MJ, McCluskey SA (2005) Early clinical experience with a new videolaryngoscope (GlideScope) in 728 patients. Can J Anaesth 52: 191198. [Crossref]

8. Gurkowski MA, Rasch DK (1989) Anesthetic considerations for Beckwith-Wiedemann syndrome. Anesthesiology 70: 711-712. [Crossref]

9. Gupta D, Khurana H, Kumar Sharma S, Nirwani Goyal G, Mishra S, et al. (2007) Macintosh laryngoscope aided tongue elevation eases difficult LMA classic insertion. Internet J Anesthesiol 18: 1.

10. Brimacombe J, Clarke G, Keller C (2005) Lingual nerve injury associated with the ProSeal laryngeal mask airway: a case report and review of the literature. $B r J$ Anaesth 95: 420-423. [Crossref]

Copyright: (C2015 Tan M. This is an open-access article distributed under the terms of the Creative Commons Attribution License, which permits unrestricted use, distribution, and reproduction in any medium, provided the original author and source are credited. 